\title{
DFT Comparative Study of the Formamide and Fluoro-FormamideTautomers and Their Corresponding Transition States
}

\author{
Ashraf Mansour Al-Msiedeen ( $\sim$ Ashraf_ttu@yahoo.com ) \\ Tafila Technical University
}

\section{Original Research}

Keywords: Formamide Tautomer, Fluoro-formamide Tautomer, DFT calculations, Transition State, Gibbs free energy, Enthalpy of formation

Posted Date: February 25th, 2021

DOl: https://doi.org/10.21203/rs.3.rs-164894/v1

License: (a) (i) This work is licensed under a Creative Commons Attribution 4.0 International License.

Read Full License 


\title{
DFT Comparative Study of the Formamide and Fluoro- Formamide Tautomers and Their Corresponding Transition States
}

\author{
Ashraf Mansour Al-Msiedeen \\ Ashraf_ttu@yahoo.com
}

Department of Chemistry And Technical Chemistry, Tafila Technical University, Tafila, 66110, Jordan.

\begin{abstract}
Seventeen tautomers of formamide $\left[\mathrm{NH}_{3} \mathrm{CO}\right]$ and thirty one tautomers of fluoroformamide $\left[\mathrm{NH}_{2} \mathrm{FCO}\right]$ were examined by density functional theory (DFT) calculations. Five methods of DFT (B3LYP/ 6-311++G, B3LYP/ 6-311++G(2df,2p), B3LYP/cc-pVTZ, wB97X-D/6-311++G(2df,2p) and wB97X-D/ cc-pVTZ) were used to calculate the energies and optimized geometries of formamide tautomers. B3LYP/cc-pVTZ method was determined as the best method and used to calculate relative energies, optimized geometries, mulliken charge distributions, Gibbs free energies and enthalpy of formations of all proposed fluoro-formamide/fluoroformamidic acid tautomers. Ten transition states of formamide tautomers and nineteen transition states of fluoro-formamide tautomers were determined by vibrational frequency calculations (they have an imaginary value of frequency). Using B3LYP/cc-PVTZ, the relatively small energies of formamide (A1) and formamidic acid (A6) (-446246 and $-446193 \mathrm{~kJ} . \mathrm{mol}^{-1}$, respectively ) participate to it being more stable than other formamide/formamidic acid tautomers, as well as for fluoroformamide (C1) (-706947 kJ.mol ${ }^{-1}$ ) and fluoro-formamidic acid (C3) (-706855 $\left.\mathrm{kJ} . \mathrm{mol}^{-1}\right)$. The stability of fluoro-formamide at carbonyl and at nitrogen was discussed using two thermodynamic calculations: Enthalpy of formation $[\Delta \mathrm{H}(298)]$ and Gibbs free energy $(\Delta \mathrm{G})$ values, positive $\Delta \mathrm{G}$ values (59 to $114 \mathrm{~kJ}$ mol-1) for fluoroformamidetautomerism confirms the stability of fluoro-formamide at carbonyl.
\end{abstract}

KEY WORDS: Formamide Tautomer; Fluoro-formamide Tautomer; DFT calculations; Transition State; Gibbs free energy; Enthalpy of formation. 


\section{INTRODUCTION}

Several theoretical studies have been centered on the substituents formamide tautomerism at the Nitrogen [1-12] and carbonyl carbon positions [7, 13, 14]. AM1 calculations were employed to study the impact of fluorine atom substituent at the carbonyl carbon position on the formamide tautomerism [15].The stability of rotational transition ( staggered and eclipsed) conformers of fluoro-formamide at carbon atom was determined [13]. Effect of fluorine on the reaction mechanism of proton shift in formamide was studied using MP2 method [15].This study is focused on the geometry stability of fluoro-formamide and one conformer of fluoroformamidic acid and their transition states.

We have used the DFT method for calculation studies of a series of tautomersims described in a previous report $[16,17,18]$.In this study, the possible tautomers of formamide and fluoro-formamide and their transition states are elaborated using quantum mechanical density functional theory (DFT). DFT results are used to study the stability of all tautomers and transition states at both the carbonyl carbon and at the nitrogen position, as well as details of their relative energies. A comparative study of the geometrical parameters is made between both the more stable formamide tautomers and the more stable fluoro-formamide tautomers. Mulliken charge distributions, Gibbs free energy and heat of formation of the more stable fluoroformamide tautomers were discussed.

\section{CALCULATION METHODS}

Both formamide and fluoro-formamide and their corresponding tautomers have been studied using density functional theory (DFT); the DFT/B3LYP and wB97X-D calculations were performed using Spartan 18. 6-311++G, 6-311++G(2df,2p), ccpVTZ, 6-311++G(2df,2p) and cc-pVTZ basis sets in the gas phase were used to perform geometries and energies of the all tautomers [19-25]. Transition states of formamide and fluoro-formamide tautomers were detected using the vibrational frequencies. Mulliken charge distributions, Gibbs free energy and heat of formation of the all minima fluoro-formamide tautomers have been identified using B3LYP/ccpVTZ method. 


\section{RESULTS AND DISCUSSION}

\section{Formamide/Formamidic acid tautomers:}

\section{Geometry Parameters}

In this research, five conformers of formamide and twelve conformers of formamidic acid were proposed, optimized and applied to frequency calculations. Geometrical optimizations of the more stable formamide tautomer (A1) and formamidic acid tautomer (A6) were carried out in this work by five methods of DFT [B3LYP/ 6311++G, B3LYP/ 6-311++G(2df,2p), B3LYP/cc-pVTZ, wB97X-D/6$311++\mathrm{G}(2 \mathrm{df}, 2 \mathrm{p})$ and wB97X-D/ cc-pVTZ]. Bond lengths and angles of both tautomers obtained in this work (Table I) are compared with that obtained by several theoretical and experimental methods.

As shown in Table I, the calculated values by the five methods are closed to the observed values. The minimum energies for all tautomers were obtained using the B3LYP/cc-pVTZ method (Table II) which is used to determine the percentage of error. The percentage of error $(\Delta \%)$ related to bond lengths between this work, Microwave and X-ray studies are between $(0.2-0.8) \%$ and $(0.3-1.0) \%$, respectively. On the other hand, percentage of error $(\Delta \%)$ related to angles between this work, Microwave and X-ray studies are between $(0.4-2.4) \%(0.3-1.0) \%$, respectively. This result suggests a good agreement between the calculations in this work and experimental studies especially with microwave study. As for the formamidic acid, both bond lengths and angles are close to that obtained theoretically.

\section{Relative Energies}

Methods of DFT were used to calculate the energies of all tautomers in order to determine the most stable ones. It is also used to compare the results with previous studies and with fluoro-formamide tautomersim.

Taoutomers (A1, A2, A3, A4, A5, A6 and A7 ) (Fig.1) were minima structures (have real and positive calculated vibrational frequencies). On the other hand, B1, B2, B3, B4, B5, B6, B7, B8, B9 and B10 (Fig. 2) tautomers were transition states (have an imaginary value of frequency), these results agree with those found by Min et al. [27]. 
As shown in Table II, the most stable tautomers are A1and A6, and this result corresponds with that found by experimental [25] and theoretical studies [14].According to B3LYP/cc-PVTZ method, formamide tautomer (A1) has a heat of formation $[\Delta \mathrm{H}(298 \mathrm{~K})]=-446246 \mathrm{~kJ}^{-\mathrm{mol}^{-1}}$ which is less than that in formamidic acid (A6) $\left(-446193 \mathrm{~kJ} \cdot \mathrm{mol}^{-1}\right)$.Therefore, formamide tautomer (A1)is more stable than formamidic acid tautomer. This result can be confirmed by charge distribution on the atoms of both tautomers (Scheme 1).

Charge distribution shows that the electrostatic attraction between atoms has larger magnitude (0.333) in formamide (A1) while in formamidic acid (A6) is (0.04).

\section{Fluoro-Formamide/Fluoro-Formamidic acid tautomers:}

\section{Geometry Parameters}

The B3LYP/cc-PVTZ method is the best of the five methods that have been used for formamide tautomerism as shown in previous part. Therefore this method will be employed in the study of fluoro-formamide tautomersim. Twelve conformers of fluoro-formamide and nineteen conformers of fluoro-formamidic acid were proposed, optimized and applied to frequency calculations to distinguish between tautomers and transition states. Using the B3LYP/cc-PVTZ to calculate all conformers, only twelve tautomers (Fig. 3) have real and positive calculated vibrational frequencies and nineteen corresponding transition states (Fig. 4).

Molecular geometry of the stable tautomers of fluoro-formamide molecule ((C1 and C3) based on B3LYP/cc-PVTZ method were calculated, bond lengths and bond angles of the two tautomers are shown in Table III.

Geometry parameters of tautomers $\mathrm{C} 1, \mathrm{C} 3, \mathrm{~A} 1$ and A6 were compared based on B3LYP/cc-PVTZ method. By comparison in (Tables II and III) we can see that (bond lengths and angles) between ( $\mathrm{C} 1$ and $\mathrm{A} 1)$ and (A6 and $\mathrm{C} 3$ ) were affected by fluorine substituent on the carbonyl carbon for two parent conformers (A1 and A6). The bond lengths of formamide (A1) were slightly affected by fluorine substituent and the most obvious bond length on the parent conformer (A1) was $\mathrm{C}=\mathrm{O}$ bond $(0.02 \AA)$. 
According to bond angles, the $\mathrm{O}_{6}-\mathrm{C}_{4}-\mathrm{N}_{2}$ was the most affected bond angle which is increased by 4.05 degrees. As for the effect of the presence of fluorine atom - on the carbon of formamidic acid (A6) - the affected bond was C1- N3 where length was decreased by $0.015 \AA$. Regarding to the bond angles, we can see the new arrangement of atoms that made by substitute fluorine (C3), where the angle $\mathrm{O}_{2}-\mathrm{C}_{1}-\mathrm{N}_{3}$ has decreased 2.24 degrees.

\section{Relative Energies}

Depending on the calculated results, four keto-conformers $(\mathrm{C} 1, \mathrm{C} 2, \mathrm{C} 9, \mathrm{C} 12)$ and eight enol-conformers (C3, C4, C5, C6, C7, C8, C10, C11) were detected and arranged in the Table IV from the highest stability to the lowest stability. The most stable keto and enol tautomers areC1 and $\mathrm{C} 3$ with zero-point corrected energy, $\mathrm{H}(0 \mathrm{~K})=-$ $706847 \mathrm{~kJ} \mathrm{~mol}^{-1}$ and $-706755 \mathrm{~kJ} \mathrm{~mol}^{-1}$, respectively. This result can be confirmed by the highest opposite charges in magnitude compared to other keto-conformers (Fig. 3).The amount of opposite charges of $\mathrm{C} 3$ atoms are exactly equal to that of $(\mathrm{C} 4, \mathrm{C} 5$, C6, C7) because $\mathrm{C} 3$ has two attractive forces between F-H4 and O-H6.

\section{Transition States}

Vibrational frequency calculations that obtained in this study confirm that nineteen tautomers (Fig. 4) are transition states because they have imaginary values of characteristic frequency. Self-consistent field energy, zero-point correction internal energy, enthalpy of formation and Gibbs free energy are values used in Table V.The eight transition states which achieve the most stable fluoro-formamide / fluoroformamidic acid tautomerization, are keto- tautomer (D1, D2, D3) and enol-tautomers (D9, D10, D11,D12, D13).

\section{Thermodynamic Calculations}

\section{Gibbs free energy}

All possible tautomerisms between the most stable tautomers are summarized in (Table VI). Negative Gibbs free energy values represent the forward direction to form more products (spontaneous process). On the other hand, positive Gibbs free energy represents non-spontaneous process [30]. Fluorine at carbon atom prefers the reverse 
direction that determines the stability of fluoro-formamide tautomers, while the fluoro-formamide tautomerization at nitrogen represents spontaneity process. This can be noticed by the positive $\Delta \mathrm{G}$ values (59 to114 $\mathrm{kJ} \mathrm{mol}^{-1}$ ) which are higher in magnitude. The higher values detect the stability of fluoro-formamide (at carbonyl) whereas the negative values $\left(-4\right.$ to $-33 \mathrm{~kJ} \mathrm{~mol}^{-1}$ ) of fluoro-formamide at nitrogen represent less stability.

\section{Enthalpy of formation}

When Enthalpy of formation is more negative a more stable tautomer is made [27]. Table IV shows the higher negative values of enthalpy of formation $[\Delta \mathrm{H}(298 \mathrm{~K})]$ of the more stable keto and enolfluoro-formamide tautomers; C1 (-706834 kJ.mol $\left.{ }^{-1}\right)$ and C3 (-706742 kJ.mol $\left.{ }^{-1}\right)$, respectively.

\section{CONCLUSION}

The B3LYP/cc-pVTZ method is considered a suitable method for the calculation of geometry as the values computed in this way correspond to the observed values. In addition, the lowest energies of all formamide tautomers were obtained through this method. Therefore, we relied on this method in our calculations of fluoro-formamide tautomersim. The proper tautomerization of fluoro-formamide / fluoro-formamidic acid is between the more stable tautomers which areC1 (keto-tautomer) and C3 (very narrowly from $\mathrm{C} 4)$ (enol-tautomer). For the most stable tautomers ( $\mathrm{C} 1$ and $\mathrm{C} 3)$, there are two possible paths: 1- From left to right where the energy needed for this direction ranged from 91.58 to $425.82 \mathrm{~kJ} \mathrm{~mol}^{-1}$, 2- from right to left where the energy needed ranged from 43.55 to $545.61 \mathrm{~kJ} \mathrm{~mol}^{-1}$. Difference in stability of all fluoro-formamide tautomers is due to the attractive and repulsive forces variation within each conformer (Fig. 3), discussing the stability of $\mathrm{C} 1$ and $\mathrm{C} 3$ tautomers (Scheme 2). The more nonspontaneous processes are when $\mathrm{C} 1$ is a reactant $\left(\Delta \mathrm{G}=95\right.$ to $\left.114 \mathrm{~kJ} \mathrm{~mol}^{-1}\right)$ that discussingthe stability of $\mathrm{C} 1$ tautomer. 


\section{FUNDING}

No funding was received for this theoretical article.

\section{COMPLIANCE WITH ETHICAL STANDARDS}

Conflicts of interest: The author declares that he has no conflict of interest.

\section{REFERENCES}

1. Shipman, L. L., and Christoffersen, R. E., J. Am. Chem. Soc., 1973, 95, 1408; Perricaudet, M., and Pullman, A, J. Peptide Protein Res., 1973, 5, 99.

2. Ottersen, T., Acta Chem. Scand.,1975, A29, 939.

3. Hagler, A. T., Leiserowitz, L., and Tuval, M., J. Am. Chem. Soc.,1976, 98, 4600. Reference 4: Peters, D., and Peters, J., J. Mol. Struct., 1978, 50, 133.

4. Klimkowski, V. J., Sellers, H. L., and Schafer, L., J. Mol. Struct., 1979, 54, 299.

5. Fogarasi, G., Pulay, P., Torok, F., and Boggs, J. E., J. Mol. Struct., 1979, 57, 259.

6. Williams, J. O., van Alsenoy, C., and Schafer, L., J. Mol. Struct., 1981, 76, 171.

7. Radom, L., Riggs, N. V., Aust. J. Chem.,1982, 35 (6) 1071 - 1077.

8. Headley, A, D., Nam, J., Theochem. J. Mol. Struct.Theochem, 2002,10.1016/S0166-1280(02)00301-9, 589-590, (423-429).

9. G. Fogarasi, G., Pulay, P.,Torok, F., Boggs, J.E.,J. Mol. Struct. 57(1979) 259.

10. Robb, M. A., Csizmadia, I. G., J. Chem. Phys., 1969, 50, 1819.

11. Radom, L., Riggs, N.V.,Aust. J. Chem.,1980, 33, 249.

12. Hoesterey, B., Neely, W.C., Worley, S.D.,Chem. Phys. Lett.1983, $94,311$.

13. Kian, T. L.,Michelle M. F., J. Phys. Chem. 1987, 91, 11, 2716-2721.

14. Hamzeh S. M., ActaChim. Slov. 2009, 56, 835-844.

15. Heidar R., Mehdi Y., Int. J. Quantum Chem., 2012, 112 (11) , 2378-2381.

16. Al-Mazaideh, G. M., Ababneh, T. S., Abu-Shandi, K. H., Jamhour, R. M. A. Q., Ayaal Salman, H. J., Al-Msiedeen, A. M., Khalil, S. M., Phy. Sci. Inter. J., 2016; 12(1): 1-7.

17. Al-Msiedeen, A.M.; Al-Mazaideh, G.M.; Khalil, S.M., Chem. Sci. J., 2016, 13:18.

18. Al-Mazaideh, G. M., Al-Msiedeen, A. M., Alakhras, F., Aldal'in, H., Ayyal Salman, H. J., Al-Itiwi, Z., Al Khalyfeh, K., Khalil, S. M., J. J. E. C. I., 2018, 1. 1. 38 -44 .

19. Becke, A.D., J. Chem. Phys., 1993, 98, 5648-5652.

20. Becke, A.D., J. Chem. Phys., 1996, 104, 1040.

21. Bernard, Y. A., Shao, Y., Krylov, A., J. Chem. Phys., 2012, 136, 204103.

22. Lee, C., Yang, W., Physical review. B, 1988, 37(2), 785-789.

23. Petersson, G.A., Andrew, B., Thomas G. T., Mohammad A. A., William A. S., J. Chem. Phys. 89, 2193 (1988).

24. Petersson, G. A, Thomas G. T, Montgomery, J. A., J. Chem. Phys., 1991. 94, 9.

25. Kieron B., J. Chem. Phys., 2012, 136, 150901. 
26. Min-Hsien Liu, Cheng Chen \& Chuan-Wen Liu, Structural Chemistry, 2004, 15, 309-316.

27. Kurland, R. J.; Wilson, E. B., Jr. J. Chem. Phys. 1957, 27, 585.

28. Hirota, E., Sugisaki, R., Nielsen, C.J., Sorensen, A., J. Molec.Spectrosc.1974, 49, 251-267.

29. Stevens, E. D., Rys, J., Coppens, P.,J. Am. Chem. Soc.1978, 100, 2324-2328.

30. Laidler, K. J, Meiser, J.H., Physical chemistry, Bryan C. Sanctuary, 1995. 
Figures
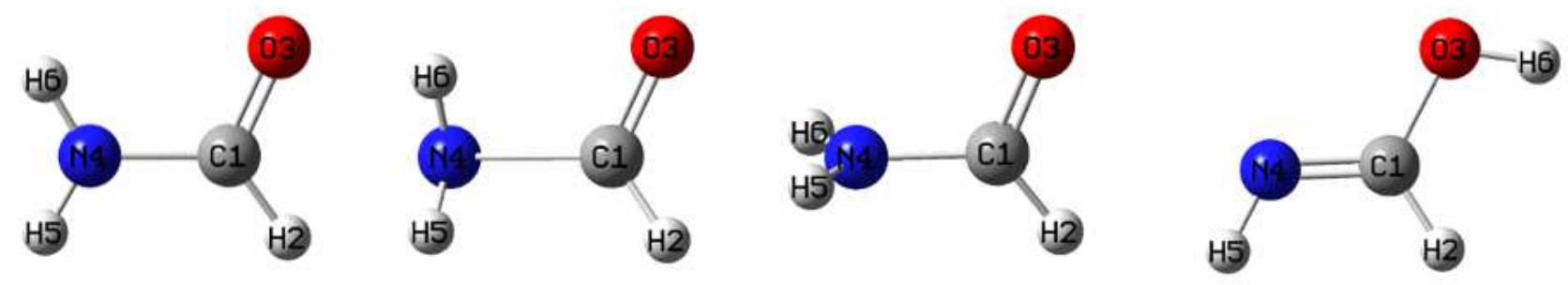

A1

A2

A3

A4
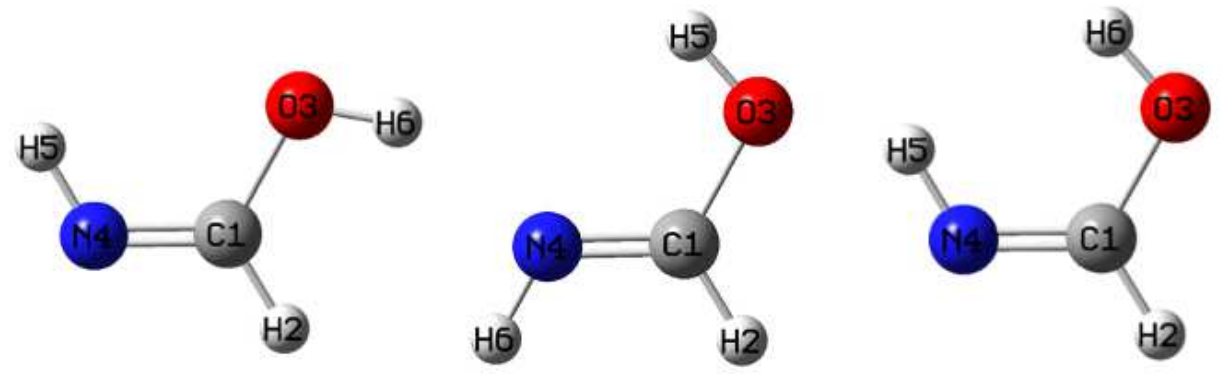

A5

A6

A7

Figure 1

Molecular geometry of the stable proposed tautomers of formamide molecule. 

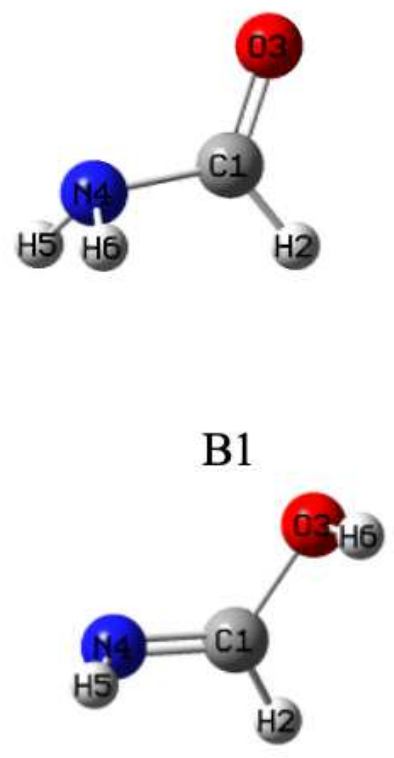

B5
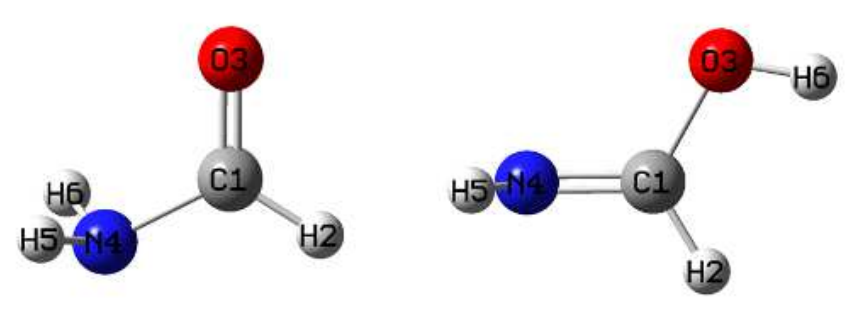

B2

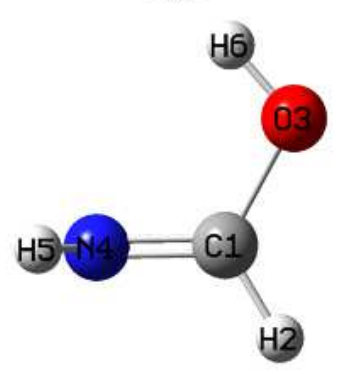

B6

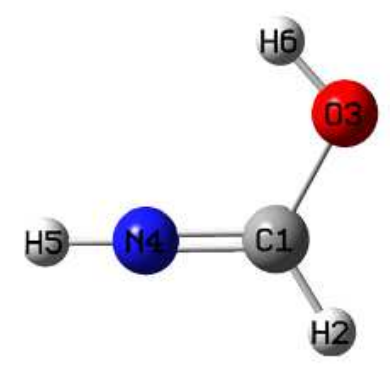

B9

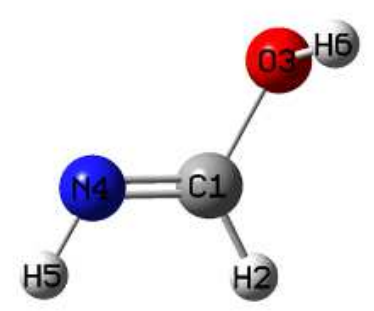

B3

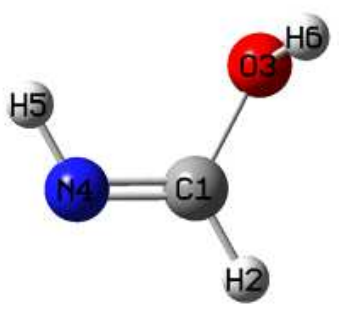

B7

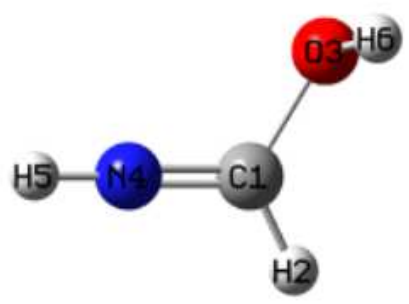

B10

Figure 2

Molecular geometry of the transition states for formamide tautomersim. 


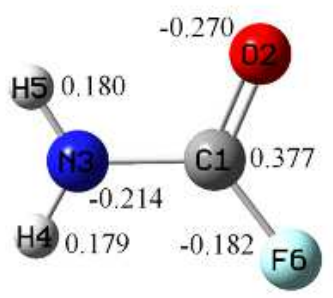

C1(-706847)

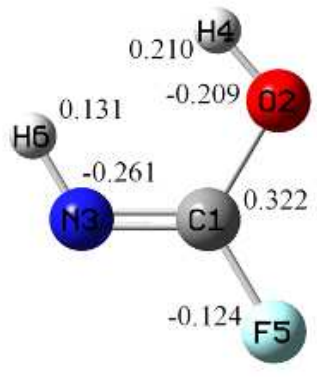

C5(-706746)

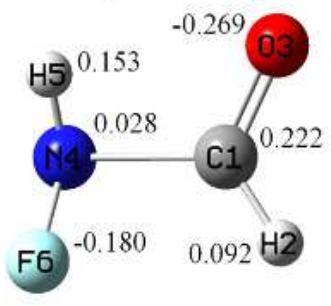

C9(-706546)

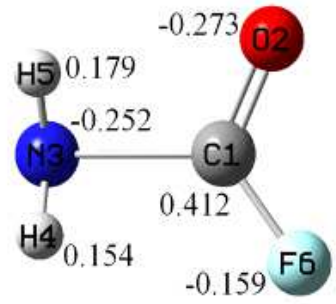

C2(-706794)

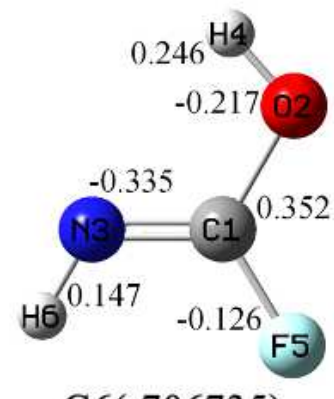

C6(-706735)

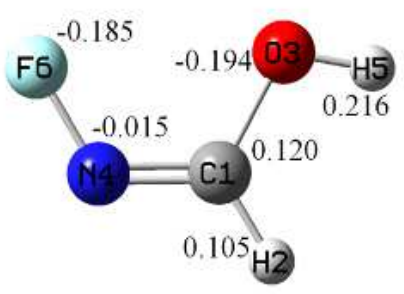

C10(-706544)

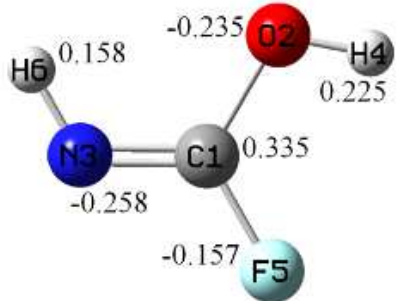

C3(-706755)

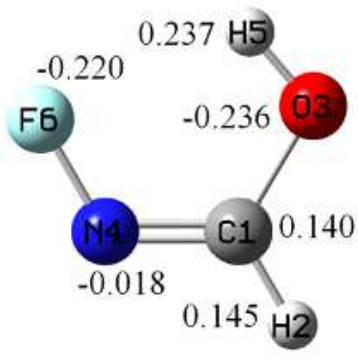

C7(-706568)

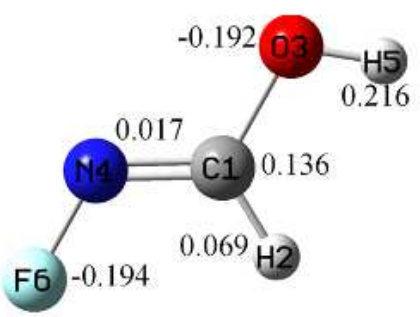

C11(-706539)

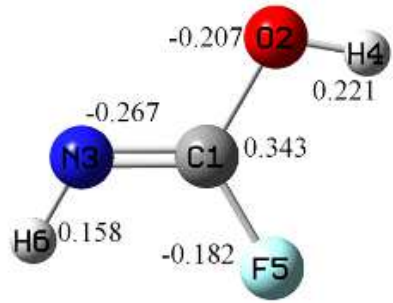

C4(-706754)
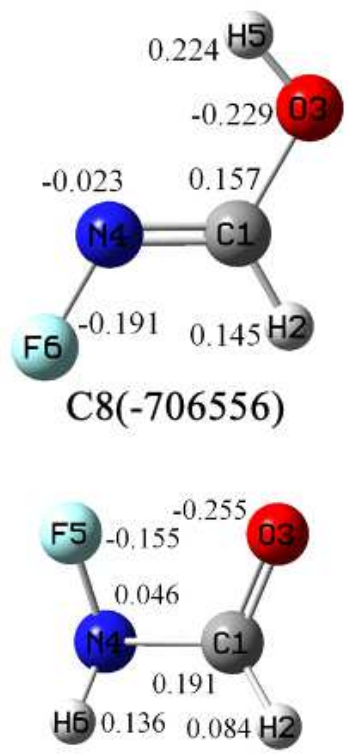

C12(-706534)

\section{Figure 3}

Molecular geometry of the stable proposed tautomers of fluoro-formamide molecule. Relative Energies (kJ.mol-1) and Mulliken Charge Distributions for fluoro-formamide tautomers (by B3LYP/cc-PVTZ method). 


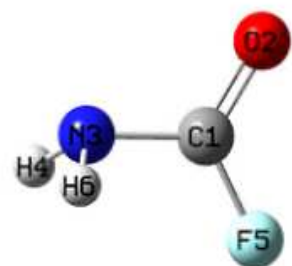

D1

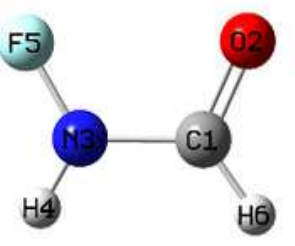

D5

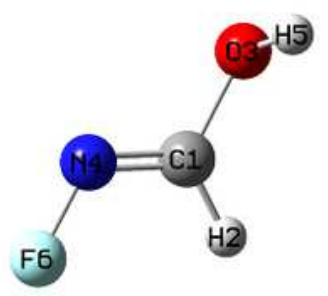

D9

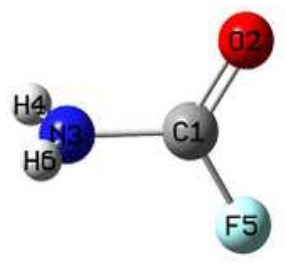

D2

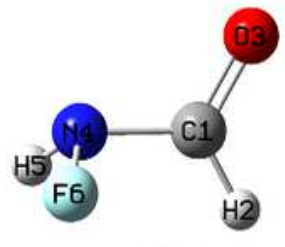

D6

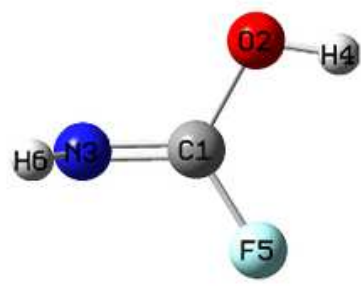

D10

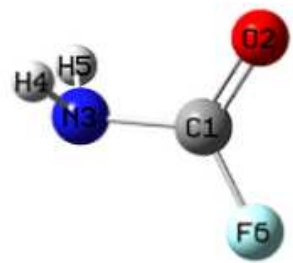

D3

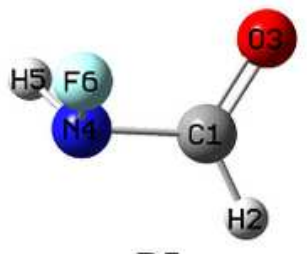

D7

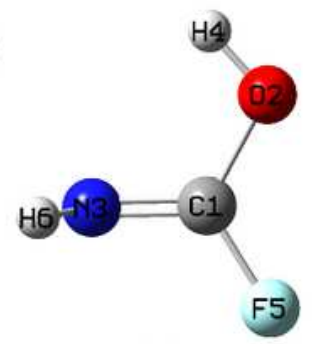

D11

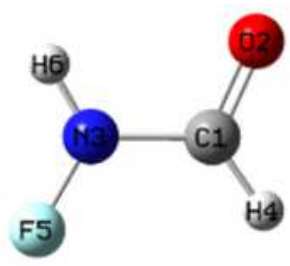

D4

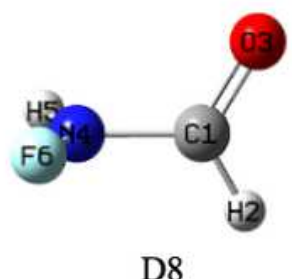

D8

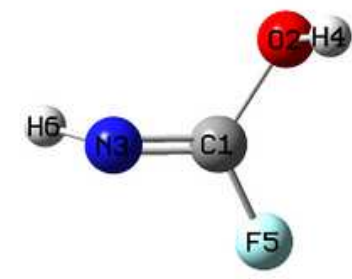

D12

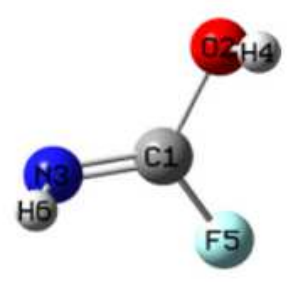

D13

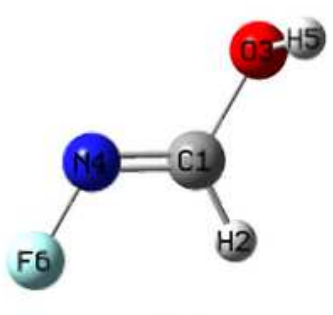

D14

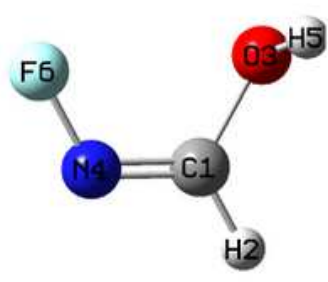

D15

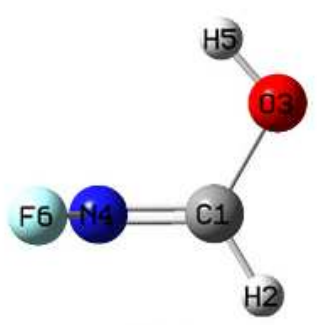

D16

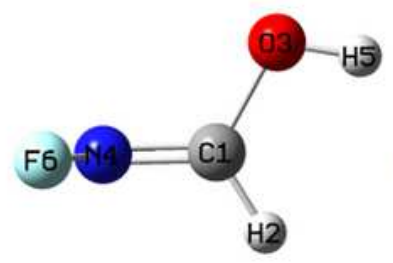

D17

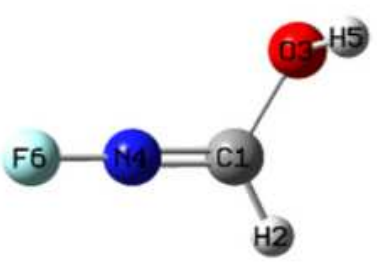

D18

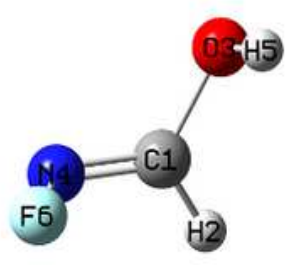

D19

\section{Figure 4}

Molecular geometry of the transition states for fluoro-formamide tautomersim.

\section{Supplementary Files}

This is a list of supplementary files associated with this preprint. Click to download. 
- Scheme1.docx

- Scheme2.docx 